\title{
Location of appendix in pregnancy: does it change?
}

This article was published in the following Dove Press journal:

Clinical and Experimental Gastroenterology

\section{Aliya Ishaq' \\ Muhammad Jamshaid \\ Husain Khan ${ }^{2}$ \\ Turab Pishori' \\ Rufina Soomro' \\ Shadab Khan ${ }^{3}$}

'Department of General Surgery, Liaquat National University Hospital, Karachi, Pakistan; ' 2 Department of Medicine, Dubai Hospital, Dubai, UAE; ${ }^{3}$ Surgical Oncology Department, Shaukat Khanum Hospital, Lahore, Pakistan
Correspondence: Aliya Ishaq

General Surgery Department, Dubai Hospital, Albaraha Street, Dubai 7272, UAE

Tel+97I 501937655

Email aishaq@dha.gov.ae
Objectives: This study aimed to determine the change in anatomical location of appendix in full-term pregnancy.

Study design: This was a descriptive cross-sectional study.

Place and duration of study: Liaquat National University Hospital, Karachi, Pakistan, Department of General Surgery, January 01 to July 31, 2010.

Patients and methods: Full-term pregnant women undergoing caesarean section were enrolled. The anatomical position of the appendix was noted by visual inspection with reference to the transtubercular plane (TTP). SPSS-10 was used for analysis.

Results: Seventy-seven full-term pregnant female patients who underwent caesarean section were included in the study. Their mean age was 29 years, the mean height was 5.3 feet, and mean gestational age was 38 weeks. Appendix was found at the normal anatomical location in 63 out of 77 patients $(81.8 \%)$, while it was located above the TTP in 14 patients $(18.2 \%)$.

Conclusion: Appendix does not migrate up with increasing gestational age in the majority of pregnant women. In most full-term pregnant female patients, appendix is located at the normal anatomical position.

Keywords: pregnancy, anatomical location of appendix, appendicitis in pregnancy

\section{Introduction}

Acute appendicitis occurs in about 1 in 500 to 1 in 635 pregnancies per year. ${ }^{1}$ Diagnostic delay is associated with significant fetal and maternal morbidity and mortality. ${ }^{2}$ Therefore, early diagnosis and prompt surgical intervention is the key to good outcome. ${ }^{3}$ This becomes challenging when a possible change in the position of the appendix with advancing pregnancy is considered. Studies done in the early 1990s prove that as pregnancy advances, the appendix migrates upward, reaching up to the level of the right hypochondrium at the end of the third trimester. ${ }^{4}$ However, new studies state that the appendix does not migrate up as pregnancy advances. ${ }^{5} \mathrm{~A}$ change in the anatomical location will change not only the presentation of patient's symptoms but also the clinical signs and site of incision for surgical intervention. Open appendectomy appears to be safe as compared to laparoscopic procedure in pregnancy. ${ }^{6}$ In this regard, the anatomical location of the appendix will have a direct impact on the choice of surgical incision.

The purpose of this study was to determine the anatomical location of the appendix in full-term pregnancy.

\section{Patients and methods Sampling technique \\ We used purposive sampling.}




\section{Study design}

The study used a descriptive cross-sectional design.

\section{Selection criteria}

Full-term pregnant women having singleton pregnancy undergoing elective and emergency caesarean (C)-section during the study period were included.

Women with maternal/fetal complications, ovarian pathology, and previous appendectomy/right hemicolectomy were excluded from the study.

The study was approved by the ethical committee of the Liaquat National Hospital. Written informed consent was taken from all patients.

\section{Data collection}

Demographic details were taken from case files, as well as by directly interviewing patients, and the data were filled in a proforma by the principal investigator.

After delivery of the baby, the location of the base of the appendix was looked for, as described in the "Operational definitions" section, and recorded in the same proforma. $\mathrm{C}$-section was performed by the on-call gynecologists, and the position of the appendix was assessed by the principal investigator.

\section{Data analysis}

All analyses were done using the Statistical Package for Social Sciences (SPSS), version 10. Mean values \pm standard deviation were computed for all quantitative variables, including patient age, gestational age (GA), height (in feet), and anatomical location of the appendix, which was the main variable of interest (categorical variable) and was calculated as percentages.

Age and height were possible confounding variables, and stratified analysis was done to control for them.

\section{Operational definitions}

\section{Common anatomical location of appendix}

The base of the vermiform appendix is attached to the posteromedial aspect of the cecum. It is located in the right iliac fossa (RIF). ${ }^{7}$ The RIF is defined as an area to the right of the vertical midline and inferior to the transverse transtubercular plane (TTP). ${ }^{8}$

\section{TTP/intertubercular plane}

It is an imaginary line drawn in the horizontal plane at the upper margin of the iliac tubercles. It is at the level of the L5 vertebra and corresponds to the upper limit of the RIF. ${ }^{9}$ It was determined after identifying the iliac tubercles (highest point on the iliac crest) by palpating the iliac crest on the skin of the abdomen. The TTP was taken as the reference line to determine the anatomical location of the appendix after delivery of the baby by $\mathrm{C}$-section. Location of the appendix was determined by visual inspection and was considered to be at the normal or common anatomical location if the appendix was located at or below this line. If the appendix was located above this line, it was considered as upward migration of the organ. The distance was measured using the finger of the researcher, which was calibrated in centimeters using a centimeter scale before each procedure.

\section{Full-term pregnancy}

Pregnancy between 37 and 40 weeks of gestation and beyond is considered full term. ${ }^{10}$ Pregnancy was confirmed by ultrasonography (done in every case preoperatively for fetal well-being), and the GA was mentioned in the case file by the gynecologist.

\section{Results}

Seventy-seven full-term pregnant female patients fulfilled the inclusion and exclusion criteria. Their mean age was 29 (19-39) years. The mean height of the patients in our study was $5.3(5.0-6.0)$ feet. The mean GA was $38.2(37-42)$ weeks (Figure 1).

The anatomical location of the appendix was found to be below the iliac crest in 47 (61\%), at the iliac crest in 16 (20.8\%), and above the iliac crest in $14(18.2 \%)$ patients.

The appendix was therefore found in its normal anatomical location, ie, at or below the TTP in 63 patients $(81.8 \%)$, while it was found at a higher position above the iliac crest in 14 out of 77 patients (18.2\%) (Figures 2 and 3).

In the 14 patients in whom the appendix migrated above the TTP, the mean upward displacement was $2.8 \mathrm{~cm}(1.0$ $4.5 \mathrm{~cm}$ ). The maximum upward displacement was $4.5 \mathrm{~cm}$. One patient had GA of 38 weeks, and the appendix was found in the right hypochondrium.

Stratified analysis of the confounding variables "age" and "height" was done.

Height was stratified in 2 groups: Group 1 (H1) between 4 and 5 feet; and Group 2 (H2) between 5 and 6 feet.

There were 48 patients in the GA1 group, having GA between 37 and 38 weeks. Of these 48 patients, 11 (22.9\%) had appendix located above the TTP, while it was located below the TTP in 37 (77.1\%) patients. In the GA2 group, there were 24 patients, 3 of whom (12.5\%) had appendix located above the TTP, while $21(87.5 \%)$ had appendix 


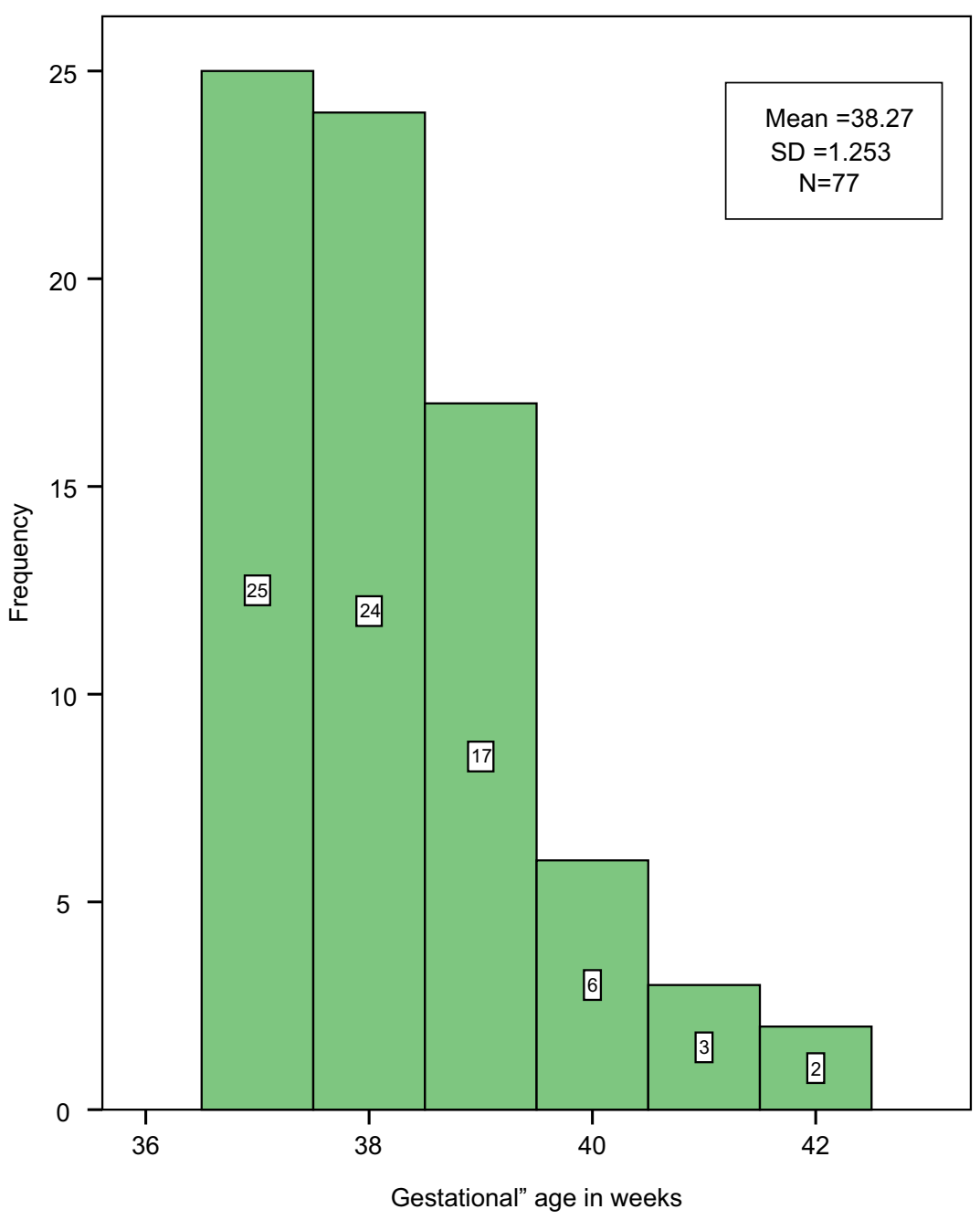

Figure I Distribution of gestational age, in weeks, in the study population.

Notes: Mean $=38.27$ weeks; $S D=1.253$ weeks; $N=77$. White box in each bar indicates the number of patients in each gestational age week. Mean gestational age $=38.2$ weeks; maximum gestational age $=42$ weeks; minimum gestational age $=37$ weeks.

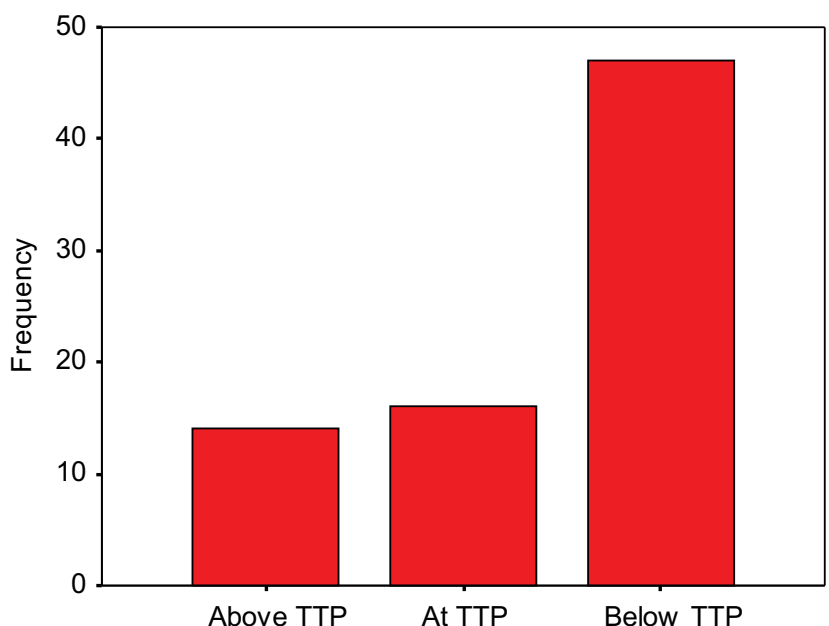

Figure 2 Anatomical location of appendix in relation to the TTP. Abbreviation: TTP, transtubercular plane.

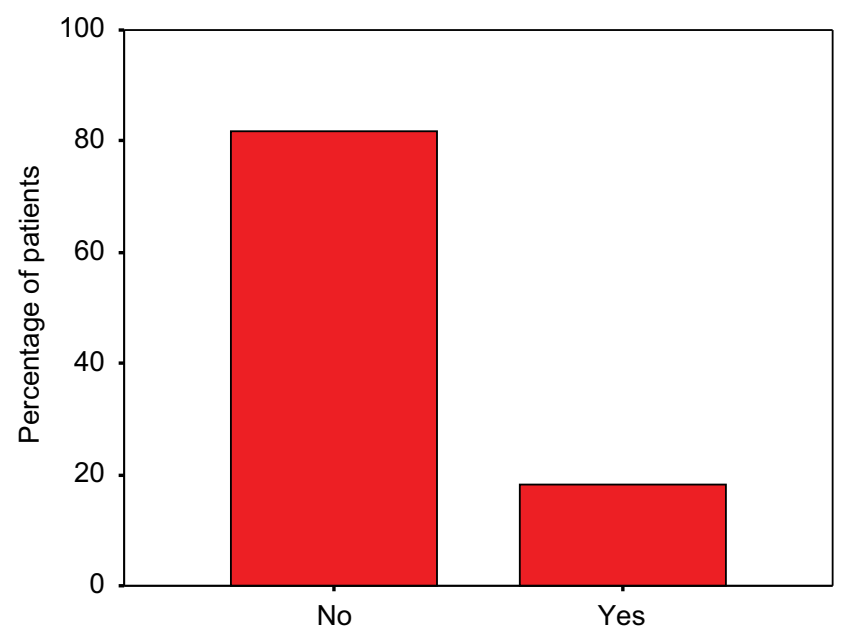

Figure 3 Percentage of patients having migration of appendix above the TTP. Abbreviation: TTP, transtubercular plane. 
at the normal anatomical location. In GA3, the group with between 41 and 42 weeks of gestation, there were 5 patients, and none of them had appendix located above the TTP (Figure 4).

\section{Discussion}

Although a rare presentation, appendicitis is one of the most common causes of an acute abdomen in pregnancy, occurring in $\sim 1$ in 500 to 1 in 2000 pregnancies. ${ }^{11}$ This represents an overall incidence of $0.05 \%-0.07 \%{ }^{12}$ and does not appear to be any different in the nongravid population. Incidence rates in the first trimester range from $19 \%$ to as high as $36 \% .^{13}$ There is a higher incidence of appendicitis in the second trimester, ranging from $27 \%$ to $60 \%$. Although the incidence decreases from $15 \%$ to $33 \%$ in the third trimester, some studies have reported a $59 \%$ incidence in the third trimester. ${ }^{14}$ Perforation rates for pregnant patients have been reported to be as high as $55 \%$ of cases, compared with $4 \%-19 \%$ in the general population. Due to the lack of specificity of the preoperative evaluation, the pathologic diagnosis of appendicitis is confirmed in only $30 \%-50 \%$ of cases. ${ }^{15}$ The risk of delay in diagnosis is associated with a greater risk of complications, such as perforation, infection, preterm labor, and risks of fetal or maternal loss. Maternal mortality has been reported from $0 \%$ to $2 \%$. An unruptured appendix carries a fetal loss of $1.5 \%-9 \%$, while this rate increases up to $36 \%$ with perforation. The risk for premature delivery is the greatest during the first week after surgery. ${ }^{16}$

Traditionally, an early exploration in suspected cases is indicated to avoid complications related to delay. A high negative appendectomy rate therefore has been accepted. Previous studies have reported a negative appendectomy rate of $11 \%-50 \%$. However, there are few high-quality data supporting the safety of this approach. Negative appendectomy in pregnant women is not risk free and exposes both mother and child to risks similar to those when surgery is performed for acute appendicitis in nonpregnant

Table I Anatomical location of appendix in relation to gestational age when gestational age is stratified

\begin{tabular}{llll}
\hline $\begin{array}{l}\text { Anatomical location of } \\
\text { appendix }\end{array}$ & GAI & GA2 & GA3 \\
Migrated above TTP & I I (22.9) & $3(12.5)$ & N=5 \\
\hline No change & $37(77.1$ & 2 I $(87.5)$ & $5(100)$ \\
\hline
\end{tabular}

Notes: GAI = GA between 37 and 38 weeks; GA2 = GA between 39 and 40 weeks; $\mathrm{GA} 3=\mathrm{GA}$ between $4 \mathrm{I}$ and 42 weeks. Data shown as $\mathrm{n}(\%)$.

Abbreviations: GA, gestational age; TTP, transtubercular plane. women. ${ }^{17}$ The scoring systems, as well as the clinical and biochemical parameters, used to diagnose appendicitis in the nonpregnant population are not very useful in the setting of pregnancy because the signs and symptoms of appendicitis, including tachycardia, low blood pressure, fever, and elevated white blood cell count, are attributed to normal pregnancy as well; therefore, careful preoperative assessment and imaging should be done to reduce the historically high negative appendectomy rates in pregnancy. ${ }^{18}$

In pregnant women with suspected appendicitis, a positive ultrasonogram (US) requires no further confirmatory test. However, in the case of nonvisualization of the appendix on US, magnetic resonance imaging (MRI) is the recommended imaging examination since it yields a high diagnostic rate and accuracy. ${ }^{19}$

Surgical intervention in pregnancy, both laparotomy and laparoscopy, has been shown to increase adverse pregnancy outcomes. Furthermore, it has been noted that pregnancy outcomes following surgery for appendicitis are worse than with surgery for other indications. Any surgeon treating a pregnant patient must have a thorough understanding of the physiology of the pregnant patient, as well as the risks and benefits of laparoscopic surgery. The possible drawbacks are injury to the uterus during Veress needle insertion, potential reduction of uterine blood flow secondary to increased intra-abdominal pressure, risk of $\mathrm{CO}_{2}$ absorption by the mother and child, and the technical difficulty of laparoscopic surgery. Physiologic and anatomic changes introduce certain risks unique to the gravid patient, some associated with laparoscopy in pregnancy. These risks have been postulated to include poor visualization due to gravid uterus, uterine injury during trocar placement, decreased uterine blood flow, or premature labor from the increased intra-abdominal pressure and increased fetal acidosis or other unknown effects due to $\mathrm{CO}_{2}$ pneumoperitoneum. Fetal hemodynamic abnormalities (tachycardia and hypertension) are also attributed to fetal hypercarbia and can be reversed by mild maternal respiratory alkalosis. Monitoring maternal arterial blood gases has proven superior to maternal capnography. ${ }^{20}$

Although the laparoscopic approach to appendectomy in pregnancy is associated with a low rate of intraoperative complications in all trimesters, the rate of fetal loss following laparoscopic appendectomy is almost $6 \%$, which is significantly higher than that following open appendectomy. Rates of preterm delivery would appear to be equal or slightly better in the laparoscopic appendectomy group. It would appear, therefore, that pregnant women requiring appendectomy 
should undergo an open procedure in the interests of fetal well-being. ${ }^{19}$ It is regrettable that much of the available data on laparoscopic appendectomy in pregnancy are derived from case reports and retrospective case series. However, given the surgical expertise needed to confidently perform laparoscopic procedures in pregnant women, a large randomized trial to address the optimal surgical approach to appendicitis in pregnancy seems unlikely. ${ }^{21}$

Determining the anatomical location of the appendix in pregnant patients is very crucial as open appendectomy is the procedure of choice in pregnant women as compared to the nonpregnant population, wherein laparoscopic appendectomy is usually performed when expertise and facilities are available. ${ }^{21}$ Identifying the correct anatomical location helps in the correct placement of the incision and has subsequent positive effects on perioperative management, fetal well-being, and patient recovery.

Classical obstetrics teaching has been that the location of the appendix and hence the pain of appendicitis migrates up with a growing uterus. This concept was based on a study done by Baer et $\mathrm{al}^{4}$ in 1932 using barium enemas. The results of this study have not been replicated as pregnant women cannot be exposed to unnecessary X-rays. The findings of this study have been challenged by the results of recent clinical research studies, which show that the anatomical location of the appendix does not change as pregnancy advances. ${ }^{5}$ Therefore, this study was conducted to reevaluate the anatomical location of the appendix in full-term pregnant women undergoing C-section.

The results of our study show that there is no relationship between GA and anatomical location of the appendix. According to our results, $22.9 \%$ patients (11/48) with GA between 37 and 38 weeks, 12.5\% (3/24) patients with GA between 39 and 40 weeks, and $0 \%(0 / 5)$ patients with GA of 41-42 weeks showed upward migration of the appendix. In our study, the single patient who had maximum upward migration of the appendix ( $4.5 \mathrm{~cm}$ above the TTP) showed a GA of 38 weeks. The results of our study are in contradiction to the results from other studies showing upward migration of the appendix with increasing GA. ${ }^{4}$ The results of our study can be compared with those from the study by Popkin et al, who found that there was no relationship between GA and location of the appendix. ${ }^{22}$

The main outcome of interest in our study was the anatomical location of the appendix. Appendix was found at the normal anatomical position (at or below the iliac crest) in 63 out of 77 patients, which accounts for $81.8 \%$ of patients. Appendix was found higher up in 14 out of 77 patients, which equals $18.2 \%$ of patients. The average upward migration was $3 \mathrm{~cm}$ above the iliac crest, with a range from $1 \mathrm{~cm}$ to $4.5 \mathrm{~cm}$. The results of our study show that the appendix does not migrate upward in most of the pregnant women as pregnancy advances; rather its location remains in the RIF regardless of the GA. The results of our study can be compared with those of the study by Hodjati and Kazerooni, ${ }^{5}$ which showed that the appendix migrates up in only $15 \%$ of pregnant women undergoing C-section, while in $85 \%$ of cases, it was located at the normal anatomical location. The mean upward displacement in this study was 3-4 cm from McBurney's point. This value is close to the results of our study.

We used the tool of visual inspection to look for the anatomical location of the appendix in pregnant women, as used by Hodjati and Kazerooni ${ }^{5}$ and Popkin et al. ${ }^{22}$ Both these studies and our study showed that upward migration of the appendix in pregnant women is not significant, while Baer et $\mathrm{al}^{4}$ and Oto et $\mathrm{al}^{23}$ studied the location of the appendix in pregnant women roentgenologically and found significant upward migration. This observation raises a question regarding the possibility of finding less upward displacement in pregnant women when the appendix is observed after opening up the peritoneal cavity. Theoretically, one can think that once we open up the abdomen, we increase the space in the peritoneal cavity, as compared to the closed cavity when examined roentgenologically. Furthermore, to look for the appendix, we apply retractors to create more space, and this step further increases the space in the abdominal cavity and can result in the downward displacement of the appendix. In our study, we examined the appendix after the baby was delivered, and this reduces the size of the uterus and theoretically can provide further space and can lead to further downward displacement of the appendix. This can be one of the reasons that our study does not show significant upward displacement of the appendix. But if this is considered true, then there are still $18.2 \%$ cases in our study showing mean upward migration of $3 \mathrm{~cm}$ above the iliac crest/TTP; moreover, in our study, there was 1 patient who had the appendix located $4.5 \mathrm{~cm}$ above the iliac crest, which corresponds to the right hypochondrium, despite all the earlier-mentioned factors. There is 1 more point in the study by Baer et al, ${ }^{4}$ and it says that the appendix returns to normal 9 days postpartum; if we take Baer's observations as true, it means that evacuation of the uterus does not have immediate effect on the location of the appendix. So, 
keeping in mind all these facts, it seems that opening up the abdominal cavity, using retractors to increase the space in the peritoneal cavity, and evacuating the uterus do not produce immediate effects on the anatomical location of the appendix and, hence, the appendix cannot be displaced by all these factors.

\section{Conclusion}

In our observations, the anatomical location of the appendix does not change in most full-term pregnant women, and increasing GA does not cause the appendix to migrate up, above the normal anatomical location. But, this is a descriptive cross-sectional study without any control group, and the sample size is also very small, so the results cannot be generalized. This study can provide a base for future studies with a control group and a much larger population for evaluation of the real location of the appendix in pregnancy, so that results can be generalized.

A high level of clinical suspicion is needed to quickly and correctly diagnose appendicitis in pregnant women. Appendicitis should be included on the differential diagnosis list for right lower quadrant pain.

\section{Acknowledgments}

We are thankful to the Department of General Surgery, Liaquat National Hospital, Karachi, Pakistan, for bearing the cost for surgical gowns, gloves, and measuring scales used for each patient. We are thankful to Dr Maryum Makki, Resident, General Surgery Department, Dubai Hospital, Dubai, UAE, for helping in the final writing with references and corrections.

Dr Turab Pishori is now working as a consultant general surgeon at Bairnsdale Regional Health Service, Bairnsdale, VIC, Australia. Dr Aliya Ishaq's current address is the General Surgery Department, Dubai Hospital, Dubai, UAE.

\section{Disclosure}

The authors report no conflicts of interest in this work.

\section{References}

1. Franca Neto AH, Amorim MM, Nóbrega BM. Acute appendicitis in pregnancy: literature review. Rev Assoc Med Bras. 2015;61(2):170-177.

2. Jung SJ, Lee DK, Kim JH, Kong PS, Kim KH, Bae SW. Appendicitis during pregnancy: the clinical experience of a secondary hospital. J Korean Soc Coloproctol. 2012;28(3):152-159.
3. Sachs A, Guglielminotti J, Miller R, Landau R, Smiley R, Li G. Risk factors and risk stratification for adverse obstetrical outcomes after appendectomy or cholecystectomy during pregnancy. JAMA Surg. 2017;152(5):436-441.

4. Baer J, Reis R, Arens R. Appendicitis in pregnancy, with changes in position and axis of normal appendix in pregnancy. JAMA. 1932;98: 1359-1363.

5. Hodjati H, Kazerooni T. Location of the appendix in the gravid patient: a re-evaluation of the established concept. Int J Gynaecol Obstet. 2003;81(3):245-247.

6. Walsha CA, Tangb T, Walshb SR. Laparoscopic versus open appendicectomy in pregnancy: a systematic review. Int J Surg. 2008;6(4):339-344.

7. Neil J, Ashraf S. The small and large intestine. In: Williams NS, Bulstrode CJK, O'connel PR, editors. Bailey and Love's Short Practice of Surgery. 25th ed. London: Hodder Arnold; 2008:1154-1155.

8. Liu C, McFadden D. Acute abdomen and appendix. In: Grennfield L, Mulholland M, Oldham K, editors. Surgery: Scientific Principles and Practice. Philadelphia: Lippincott-Raven; 1997:1246-1261.

9. Young B. Gastrointestinal tract. In: Burkitt HG, Young B, Heath JW, editors. Wheater's Functional Histology, A Text and Colour Atlas. Newcastle, Australia: Churchill Livingstone; 1993:247-271.

10. Farquharson M, Moran B. General techniques in abdominal and gastrointestinal surgery. In: Farquharson M, Moran B, editors. Farquharrson's Text Book of Operative General Surgery. 9th ed. London: Hodder Arnold; 2005:222-226.

11. Walker HG, Al Samaraee A, Mills SJ, Kalbassi MR. Laparoscopic appendicectomy in pregnancy: a systematic review of the published evidence. Int J Surg. 2014;12(11):1235-1241.

12. Machado NO, Grant CS. Laparoscopic appendicectomy in all trimesters of pregnancy. JSLS. 2009;13:384-390.

13. Mourad J, Elliott JP, Erickson L, Lisboa L. Appendicitis in pregnancy: new information that contradicts long-held clinical beliefs. Am JObstet Gynecol. 2000;182(5):1027.

14. Zingone F, Sultan AA, Humes DJ, West J. Risk of acute appendicitis in and around pregnancy: a population-based cohort study from England. Ann Surg. 2015;261(2):332-337.

15. Weston P, Moroz P. Appendicitis in pregnancy: how to manage and whether to deliver. TOG. 2015;17(2):105-110.

16. Shahid G, Dar HM, Majid HJ, Siddique Z, Mehmood S, Tufail M. A study of gynaecological emergencies presenting as acute appendicitis. Proc Shaikh Zayed Postgrad Med Inst. 2005;19(1):13-17.

17. Ito K, Ito H, Whang EE, Tavakkolizadeh A. Appendectomy in pregnancy: evaluation of the risks of a negative appendectomy. Am J Surg. 2012;204(4):145-150.

18. Gulzar S, Umar S, Dar GM, Rasheed R. Acute appendicitis - importance of clinical examination in making a confident diagnosis. PakJ Med Sci Jun. 2005;21(2):125-132.

19. Di Saverio S, Birindelli A, Kelly MD, et al. WSES Jerusalem guidelines for diagnosis and treatment of acute appendicitis. World J Emerg Surg. 2016;11:34.

20. Holzer T, Pellegrinelli G, Morel P, et al. Appendectomy during the third trimester of pregnancy in a 27-year old patient: case report of a "near miss" complication. Patient Saf Surg. 2011;5:11.

21. Palanivelu C, Rangarajan M, Parthasarathi R. Laparoscopic appendectomy in pregnancy: a case series of seven patients. JSLS. 2006;10(3): 321-325.

22. Popkin CA, Lopez PP, Cohn SM, Brown M, Lynn M. The incision of choice for pregnant women with appendicitis is through McBurney's point. AJS. 2002;183(1):20-22.

23. Oto A, Srinivisan PN, Ernst RD, et al. Revisiting MRI for appendix location during pregnancy. AJR Am J Roentgenol. 2006;186(3):883-887. 
Clinical and Experimental Gastroenterology is an international, peerreviewed, open access, online journal publishing original research, reports, editorials, reviews and commentaries on all aspects of gastroenterology in the clinic and laboratory. This journal is included on PubMed. The manuscript management system is completely online and includes a very quick and fair peer-review system, which is all easy to use. Visit http://www.dovepress.com/testimonials.php to read real quotes from published authors.

Submit your manuscript here: https://www.dovepress.com/clinical-and-experimental-gastroenterology-journal 